\title{
Energy Drink Consumption and its Relationship to Risky Behavior in College Students
}

\author{
Julia Buchanan and Melinda Ickes \\ University of Kentucky
}

\begin{abstract}
Background and Purpose: Energy drinks have become popular among college students, with over half reporting consumption of at least one energy drink per month in the current semester. Risk of negative physiological and psychological effects has been linked to energy drinks consumed alone or mixed with alcohol. The purpose of this study was to assess the relationship between energy drink consumption and other risky behaviors among college students at a large southeastern university in the United States. Method: A convenience sample of 277 college students was recruited during January-February 2012 via e-mail through undergraduate academic courses. Participating students completed an online survey that assessed energy drink consumption (with and without alcohol) and participation in other risky behaviors. Results: Energy drink users participated in risky behaviors more often than non-energy drink users within the past year $(p=0.00)$. Energy drink use was significantly related to the risky behaviors of tobacco $(p=$ $0.00)$, marijuana $(p=0.00)$, amphetamine $(p=0.00)$, and alcohol $(p=0.02)$ use, as well as missing class $(p=0.05)$, performing poorly on a test or important project $(p=0.05)$, and participating in an extreme sport $(p=0.03)$. Conclusion: These findings suggest that energy drink consumption is associated with other risky behaviors among college students. The benefits of health promotion efforts related to energy drink consumption may be furthered through co-occurrence with other education and prevention programming on the topics of substance use and abuse.
\end{abstract}

(c) 2015 Californian Journal of Health Promotion. All rights reserved.

Keywords: energy drinks, risk taking, risky behavior, college students, college health promotion

\section{Introduction}

An increasingly problematic issue among college students is the excessive consumption of energy drinks, both with and without alcohol (EDs). Since the 1997 debut of Red Bull, the consumption of energy drinks without alcohol (EDNAs) has risen among this population (Miller, 2008). Caffeinated alcoholic beverages, beverages that are sold pre-mixed with alcohol, caffeine, and other stimulants, saw a 67-fold increase in sales from 2002 to 2008. Prompted by letters from 18 Attorney Generals and one city attorney expressing concerns regarding this mixture, the U.S. Food and Drug Administration (FDA) conducted a scientific review and concluded that caffeine "can mask some of the sensory cues individuals might normally rely on to determine their level of intoxication" leading to riskier behaviors and potentially life- threatening situations (FDA, 2010, n.p.). In November 2010, the FDA stated that these products could not stay on the market in their current form and added that caffeine is an unsafe food additive when mixed with malt alcoholic beverages. Subsequently, seven manufacturers removed caffeine and other stimulants from their products (Centers for Disease Control and Prevention [CDC], 2014). This has not prevented the ability to mix EDNAs with alcohol, a popular behavior among college students that brings about a variety of detrimental physiological and psychological effects (Arria \& O’Brien, 2011).

\section{Prevalence}

Few studies have investigated the ED consumption habits among college students. Attila and Cakir (2011) found that $48.3 \%$ of college students reported ever trying an EDNA, 
while Malinauskas, Aeby, Overton, CarpenterAeby, and Barber-Heidal (2007) found that 51\% of college students reported consuming more than one EDNA each month in an average month for the current semester.

In regard to energy drinks mixed with alcohol (EDAs), Malinauskas et al. (2007) found that $73 \%$ of EDNA users also consumed an EDA during the past month. O’Brien, McCoy, Rhodes, Wagoner, and Wolfson (2008) reported that one-fourth of past 30-day alcohol drinkers consumed at least one EDA in the past month. Miller (2008) had a similar finding, and discovered that $26 \%$ of university students reported consuming an EDA in the past 30 days.

\section{Effects of EDNAs}

Because the rates of production, marketing, and use of EDNAs have risen so greatly in the past decade, many ( particularly college) students are simply not aware of the various risks that consumption of these beverages is associated with (Miller, 2008). The main ingredient of concern in EDNAs is caffeine - which causes negative short-term effects including dehydration (from the diuretic effect it carries). Longer term effects include reduction in insulin sensitivity, increases in mean arterial blood pressure, chronic headaches, and the possibility for central nervous system, cardiovascular, gastrointestinal and renal dysfunction (Malinauskas et al., 2007).

The FDA limits caffeine content in soft drinks to 71mg/12oz. serving size, according to the Center for Science in the Public Interest (2012). In EDNAs, however, caffeine content can vary greatly due to lack of regulation by the FDA. For the same 12 oz. serving size, common EDNAs have much greater caffeine levels; brands including Red Bull and Rockstar contain $120 \mathrm{mg}$ and $180 \mathrm{mg}$ of caffeine, respectively. Caffeine content also varies depending on container size, as some products may contain multiple servings, thus proportionally increasing caffeine levels. Furthermore, added ingredients in EDNAs (such as Guarana) also contain high amounts of caffeine, but are not listed as such on product labeling (Smith \& Atroch, 2010).
Therefore, EDNAs may have even greater caffeine levels than the consumer may perceive.

\section{Effects of EDAs}

The behavior of consuming EDAs brings about many associated dangers and risks to both the consumer and those around him or her. Clinical studies have shown that ingesting caffeine, a central nervous system stimulant, with alcohol, a depressant, reduces one's perceptions of alcohol-induced impairment compared to consuming alcohol alone (Thombs, O’Mara, Tsukamoto, Rossheim, Weiler, Merves, \& Goldberger, 2008). As a result, the user may be more prone to alcohol-related harm which includes alcohol poisoning, physical injury, impaired driving, and sexual victimization (Thombs et al., 2008). College students report consuming EDAs for reasons including a reduction in sleepiness and an increase in the pleasure sensation, which suggests that these beverages might reduce the depressant effects and/or increase the excitatory effects of alcohol (Ferreira et al., 2006). It is possible that the caffeine in an EDA could reduce the subjective feelings of being drunk (Arria \& O’Brien, 2011). Thus, those who consume these highly caffeinated beverages with alcohol may pose harm to themselves and others through the actions which result.

\section{Energy Drink Use and Risky Behavior}

In general, there is evidence to suggest that college students tend to engage in various risky behaviors, including substance abuse, violence, and eating disorders (Ahearn, 2009). For example, $80 \%$ of college students report consuming alcohol (National Institutes of Health, 2013), 44.7\% of students report partner or non-partner violence (Forke, Myers, Catallozzi, \& Schwarz, 2008), and 20\% of college women and $10 \%$ of college men struggle with an eating disorder (National Eating Disorders Association, 2013). Participation in such risky behaviors is facilitated by increased stress and newfound independence during the college years.

There is a dearth of evidence investigating the relationship between ED consumption and risky behaviors typically reported by college students. 
In one such study, EDNA consumption rates were positively associated with marijuana use, sexual risk-taking, fighting, seatbelt omission, and taking risks on a dare in college students (Miller, 2008), but there is a need to further understand these relationships. It is widely known that EDs pose physiological threats to the user's health, but risky behaviors potentially resulting from consumption (e.g., driving while intoxicated, aggressive behavior) may be cause for greater public health concern.

There is an existing gap in the literature relating ED consumption to risky behaviors, and to the authors' knowledge, no published studies exist which analyze the behavior of mixing and consuming ENDAs with alcohol among college students. Considering the heightened increase of risk-taking during adolescence (Steinberg, Albert, Cauffman, Banich, Graham, \& Wooland, 2008), it is possible that present engagement in risky behaviors, such as ED use, may influence participation in other risky behaviors (Zuckerman, 1979). Therefore, the purpose of the present study was to assess the relationship between ED consumption and other risky behaviors among college students.

In order to properly evaluate current study participants' reasoning for using EDNAs and EDAs, and subsequently comparing it to the findings of previous research, the two groups were examined individually. However, it should be noted that for the quantitative analysis exploring the relationship between these substances' use and risky behavior, the two groups were combined. Thus, ED users represented those who consumed EDNAs or EDAs at least 6 times within the past year. If each individual group of users (EDNA and EDA) would have been used, too small of a sample size would have been elicited. In addition, many of those considered to be EDNA users also consumed the beverage with alcohol, thus being representative of both substance groups. Regardless, the resulting knowledge showed a significant relationship between ED use and risky behavior. It should also be noted that knowledge gained from this study paves the way for future research on the topic. This and results of the study will be discussed in the following sections.

\section{Methods}

\section{Study Design}

A cross-sectional research design was utilized.

\section{Sample}

A convenience sample of 277 college students, attending large public southeastern university in the United States, was recruited to participate during the spring 2012 semester. The students were enrolled in activity classes and general education courses during that semester. Students enrolled in these courses were chosen because they represent a wide range of student demographics, including gender, year in school, and academic major.

\section{Measures}

The CORE Survey was used as a framework to develop a 54-item measure for the purpose of this study. The CORE Survey is a validated 39item survey developed by the Core Institute at Southern Illinois University-Carbondale to evaluate the nature and scope of alcohol and drug use on college campuses (Core Institute, 2012). In addition, the CORE Survey assesses students' attitudes, perceptions, and opinions regarding alcohol and drug use, and inquires about current and past participation in other risky behaviors (Core Institute, 2012). For a participant to be considered as having 'used' or 'engaged in' other risky behaviors, he or she must have reported doing so at least once within the past year. A sub-score based on the number of risky behaviors participants reported was calculated, ranging from 0 to 23 .

In order to meet the needs of this research study, additional questions were added regarding EDs, including rate of consumption, where students purchase the beverages, and a measure of risk awareness pertaining to ED consumption. From the original long-form of the CORE Alcohol and Drug Survey, questions 1-9, 11, 14-18, 20, and 30-34 remained unchanged and provided valuable information related to the current study's purpose. Questions 10, 12-13, 19, 22-25, 27-28, 30-32, and 37-29 were omitted, due to a 
lack of relevance to the current study's research aims. In addition, some questions were modified in order to be more specific to consumption rates of, risk awareness of, and attitudes toward EDNAs as well as EDAs. These questions from the original CORE Alcohol and Drug Survey were 16, 17, 21, 26, 29, and 35-36.

The final instrument used in this study included 52 items which were reviewed by a panel of experts to ensure content related validity. In total, 13 questions were included on participant demographics, 5 questions on ED consumption habits, 4 questions related to participant attitudes toward ED consumption, 3 questions related to influence of peers and family, and 2 questions related to availability of EDs. In addition, 2 questions were added regarding participants' behavioral intention to consume the beverages. The aforementioned alterations and additions were necessary in order to examine the relationships between consumption of these beverages and risky behaviors, risk awareness, and behavioral intention.

\section{Data Collection}

The University Institutional Review Board of the primary investigator and contributing author approved the study. An e-mail was sent to a convenience sample of 107 academic course instructors during January and February 2012, which asked them to forward the survey invitation to their enrolled students. Instructors were sent a follow-up email two weeks after the initial survey was sent, as a reminder to forward the survey. Because course instructors were not required to send their students the initial survey invitation, a definite number of students who received the e-mail was unclear. However, the potential sample size was estimated to be 1,926 (assuming an 18:1 faculty to student ratio at this university). Therefore, the estimated response rate was $14.4 \%$. An incentive was offered to students to complete the survey, which was the chance to win one of two \$25 VISA gift cards.

Missing Data. If any participant had more than $20 \%$ of the total responses considered missing, their data were eliminated from the study. In total, 14 students were eliminated from this study due to substantial missing data.

\section{Data Analysis}

Data analysis was conducted using SPSS (version 20.0) statistical software (IBM, 2011). The apriori level of significance for all statistical tests was set at $\mathrm{p}<0.05$. Frequencies and percentages were calculated to summarize the sample in regards to demographics, participation in risky behaviors and history of ED consumption.

All participants were coded into one of two categories: 1) non-energy drink users (those who consumed less than 6 EDs within the past year) and 2) energy drink users (those who consumed an ED at least 6 times within the past year). Because many of the survey participants had consumed both an EDNA and an EDA (46.5\%, $n=129)$ at least 6 times within the past year, a dichotomous variable was created to facilitate meaningful comparisons. If the criterion for an energy drink user classification was set to having consumed just one beverage in the past year, the non-user population would have been very small. The response ' 6 times/year' was the next highest available response, and thus was utilized as criterion for a respondent being classified as an energy drink user.

To determine whether ED users and non-users differed on demographic variables chi-square analyses were performed. The same process was taken to determine whether ED users and nonusers differed on participation in individual risky behaviors. Differences among ED users and non-users on their risky behavior score were examined using a one-way ANOVA test.

\section{Results}

\section{Demographic Characteristics}

Respondents represented 68 different academic majors, with the most frequent majors including: Kinesiology/Exercise Science $(n=54)$, Nursing $(n=16)$, Biology $(n=15)$, Psychology $(n=$ $13)$, Education $(n=12)$, and Business $(n=10)$. A total of $58.4 \%(n=162)$ of the respondents were female, while $40.4 \%(n=112)$ were male. Nearly all $94.6 \%(n=262)$ of participants were between the ages of 18 and 25 years, with ages $19-21$ representing $60.3 \%(n=167)$ of the sample. The mean age of the sample was 21.06 
years $(S D=3.09)$. The majority of respondents identified themselves as non-Hispanic whites, with a total of $86.6 \%(n=240)$. A total of 91.3\% $(n=253)$ of students had a GPA between $2.70(\mathrm{~B}-)$ and $4.00(\mathrm{~A})$, and $56.0 \%(n=155)$ worked part-time, while $6.1 \%(n=17)$ worked full-time. The majority of participants $(65.3 \%, n$ $=181$ ) lived in off-campus housing.
Table 1 provides demographic characteristics of the sample energy drink users and non-users separately. Gender $(p=0.01)$ and student status $(p=0.02)$ were the only two demographic variables shown to be significantly related to ED use. Females were more likely and sophomores were most likely to be ED users.

Table 1.

Demographic Characteristics of Energy Drink Users $(N=129)$ and Non-Users $(N=148)$

\begin{tabular}{|c|c|c|c|c|}
\hline & nographic & $\begin{array}{l}\text { Energy Drink } \\
\text { Users n (\%) }\end{array}$ & $\begin{array}{c}\text { Non-Users } \\
\mathrm{n}(\%)\end{array}$ & $p$ value \\
\hline \multirow[t]{2}{*}{ Gender } & Male & $62(48.1)$ & 49 (33.1) & $0.01 *$ \\
\hline & Female & $66(51.2)$ & 97 (65.5) & \\
\hline \multirow[t]{5}{*}{ Age } & $17-19$ & 43 (33.3) & $42(28.4)$ & 0.82 \\
\hline & $20-22$ & $61(47.2)$ & 77 (52.0) & \\
\hline & $23-25$ & $19(14.7)$ & $21(14.2)$ & \\
\hline & $26-28$ & $5(3.9)$ & $2(1.4)$ & \\
\hline & $29+$ & $1(0.7)$ & $6(4.1)$ & \\
\hline \multirow{8}{*}{ Race/Ethnicity } & White (non-Hispanic) & $112(86.8)$ & $128(86.5)$ & 0.39 \\
\hline & Other ${ }^{1}$ & $3(2.3)$ & $7(4.7)$ & \\
\hline & Hispanic or Latino & $3(2.3)$ & $5(3.4)$ & \\
\hline & Asian or Pacific Islander & $5(3.9)$ & $2(1.4)$ & \\
\hline & Black (non-Hispanic) & $2(1.5)$ & $5(3.4)$ & \\
\hline & American Indian /Alaskan & $2(1.5)$ & $0(0.0)$ & \\
\hline & Native, Native Hawaiian & & & \\
\hline & Biracial or multiracial & $1(0.7)$ & $1(0.7)$ & \\
\hline \multirow[t]{5}{*}{ Student Classification } & Freshman & $22(17.1)$ & $26(17.6)$ & 0.57 \\
\hline & Sophomore & 34 (26.4) & 31 (20.9) & \\
\hline & Junior & $23(17.8)$ & 35 (23.6) & \\
\hline & Senior & $31(24.0)$ & 33 (22.3) & \\
\hline & Grad/Professional & $19(14.7)$ & $21(14.2)$ & \\
\hline \multirow[t]{5}{*}{ Student Status } & Other & $0(0.0)$ & $2(1.4)$ & \\
\hline & $\begin{array}{l}\text { Full-time undergraduate (12+ } \\
\text { credits) }\end{array}$ & $109(84.5)$ & 118 (79.7) & $0.02 *$ \\
\hline & $\begin{array}{l}\text { Part-time undergraduate (1-12 } \\
\text { credits) }\end{array}$ & $0(0.0)$ & $6(4.1)$ & \\
\hline & Full-time graduate (9+ credits) & $14(10.9)$ & $22(14.9)$ & \\
\hline & Part-time graduate (1-9 credits) & $5(3.9)$ & $1(0.7)$ & \\
\hline \multirow{4}{*}{$\begin{array}{l}\text { Grade Point Average } \\
\text { (GPA) }\end{array}$} & A & $42(32.6)$ & $66(44.6)$ & \\
\hline & $\mathrm{B}$ & $75(58.1)$ & $70(47.8)$ & 0.25 \\
\hline & $\mathrm{C}$ & $11(8.5)$ & $10(6.8)$ & \\
\hline & $\mathrm{D}$ & $1(0.7)$ & $2(1.4)$ & \\
\hline
\end{tabular}

${ }^{1}$ Category available for students who did not identify with race/ethnicity choices provided; race not specified. *Denotes statistically significant interaction between demographic variable and energy drink user/non-user classification $(p<0.05)$

\section{Energy Drink Consumption}

A total of $59.2 \%$ of participants reported using EDNAs at least once within the past year, and $66.4 \%$ reported ever trying an EDNA. A total of $26.7 \%$ of participants consumed an EDA at least once within the past year, while $38.2 \%$ reported having ever tried the mixture. A total of 129 individuals were classified as energy drink users (46.5\% of the sample population). All other respondents were classified as non-users $(n=$ 148, 53.4\%), having consumed an ED less than 6 times within the past year. 
Students who acknowledged they had consumed an EDNA or an EDA also listed their reasons for doing so. Those who had consumed EDNA reported they did so due to feeling tired from insufficient sleep $(54.9 \%, n=101)$, to increase overall energy ( $50.0 \%, n=92)$, to stay awake to study or finish a project $(49.4 \%, n=91)$, and to stay awake for class $(32.6 \%, n=60)$. Other reasons reported for consuming an EDNA were to stay awake to drive a car $(30.9 \%, n=57)$ and to stay awake for work $(23.3 \%, n=43)$. Those students who consumed an EDA commonly reported the following reasons for doing so: to get drunk $(50.9 \%, n=54)$, to stay awake longer for partying (33.9\%, $n=36)$, because a friend offered it $(32.1 \%, n=34)$, to drink prior to going to bars $(17.9 \%, n=19)$, and to see what would happen (effects) $(10.4 \%, n=11)$.

Table 2.

Past Year Participation in Risky Behaviors among Energy Drink Users $(N=129)$ and NonEnergy Drink Users $(N=148)$

\begin{tabular}{|c|c|c|c|}
\hline Risky Behavior & Energy Drink Users (\%N) & Non-Users (\%N) & $p$ value \\
\hline Tobacco & $47(36.4)$ & $20(13.5)$ & $0.00^{*}$ \\
\hline Alcohol & $113(87.5)$ & $112(75.6)$ & $0.02^{*}$ \\
\hline Marijuana & $48(37.2)$ & $32(21.6)$ & $0.00^{*}$ \\
\hline Cocaine & $5(3.8)$ & $1(0.7)$ & 0.07 \\
\hline Amphetamines & $14(10.8)$ & $2(1.4)$ & $0.00 *$ \\
\hline Sedatives & $4(3.1)$ & $1(0.7)$ & 0.13 \\
\hline Hallucinogens & 5 (3.9) & $7(4.7)$ & 0.73 \\
\hline Opiates & $3(2.3)$ & $0(0.0)$ & 0.06 \\
\hline Inhalants & $1(0.8)$ & $0(0.0)$ & 0.28 \\
\hline Designer drugs & $5(3.9)$ & $4(2.7)$ & 0.57 \\
\hline Steroids & $1(0.8)$ & $0(0.0)$ & 0.28 \\
\hline Other illegal drugs & $2(1.6)$ & $1(0.7)$ & 0.48 \\
\hline Missed a class & $111(86.0)$ & $116(78.3)$ & $0.05^{*}$ \\
\hline Performed poorly on a test or important project & $103(79.8)$ & $101(68.2)$ & $0.05^{*}$ \\
\hline Done something I later regretted & $91(70.5)$ & $91(61.5)$ & 0.08 \\
\hline Got into an argument or fight & $63(48.8)$ & 59 (39.8) & 0.12 \\
\hline Not worn a seatbelt while in a car & $64(49.6)$ & $71(47.9)$ & 0.65 \\
\hline Participated in an extreme sport & $44(34.1)$ & $33(22.3)$ & $0.03^{*}$ \\
\hline Been hurt or injured & $46(35.6)$ & 38 (25.6) & 0.06 \\
\hline $\begin{array}{l}\text { Rode in a car with someone who was driving under the } \\
\text { influence }\end{array}$ & $47(36.4)$ & $43(29.0)$ & 0.16 \\
\hline Driven a car while under the influence & $28(21.7)$ & $24(16.2)$ & 0.19 \\
\hline $\begin{array}{l}\text { Been in trouble with police, residence hall, or other college } \\
\text { authorities }\end{array}$ & $14(10.8)$ & $11(7.4)$ & 0.31 \\
\hline Experienced signs/symptoms of an eating disorder & $14(10.8)$ & $17(11.5)$ & 0.91 \\
\hline Tried unsuccessfully to stop using drugs or alcohol & $6(4.7)$ & $7(4.7)$ & 0.99 \\
\hline Seriously thought about suicide & $12(9.3)$ & $8(6.8)$ & 0.20 \\
\hline Have been taken advantage of sexually & $10(7.8)$ & $10(6.8)$ & 0.73 \\
\hline Damaged property, pulled fire alarm, etc. & $3(2.3)$ & $7(4.7$ & 0.30 \\
\hline Have taken advantage of another sexually & $2(1.5)$ & $3(2.0)$ & 0.78 \\
\hline Tried to commit suicide & $2(1.5)$ & $0(0.0)$ & 0.13 \\
\hline Been arrested for DUI/DWI & $1(0.8)$ & $1(0.7)$ & 0.20 \\
\hline
\end{tabular}

* Denotes statistically significant interaction between risky behavior participation and energy drink user/non-user classification $(p<0.05)$

\section{Participation in Risky Behaviors}

Past-year participation in individual risky behaviors is summarized in Table 2, for both energy drink users $(N=129)$ and non-users $(N$ $=148)$. For energy drink users, the most frequent responses for past-year engagement in risky behaviors were alcohol use $(87.5 \%, n=$ 113), missing a class $(86.0 \%, n=111)$, performing poorly on a test or important project (79.8\%, $n=103)$, doing something they later 
regretted $(70.5 \%, n=91)$, not wearing a seatbelt in the car $(49.6 \%, n=64)$, and getting into an argument or fight $(48.8 \%, n=63)$. For nonusers, the most frequent responses for past-year engagement in risky behaviors were missing a class $(78.3 \%, n=116)$, alcohol use $(75.6 \%, n=$ 112 ), performing poorly on an important test or project $(68.2 \%, n=101)$, and doing something they later regretted $(61.5 \%, n=91)$.

Certain risky behaviors were significantly related to ED use, and are listed in Table 2. ED users exhibited significantly higher rates of tobacco $(p=0.00)$, alcohol $(p=0.02)$, marijuana $(p=0.00)$, and amphetamine $(p=$ 0.00 ) use than non-users. In addition, ED users were more likely to have missed a class ( $p=$ 0.05 ), performed poorly on a test or important project $(p=0.05)$, and participated in an extreme sport ( $p=0.03$ ) as compared to nonusers. Comparisons were made using chi-square analyses.

Participants received a total risky behavior score based on whether or not they had used certain substances or participated in certain risky behaviors at least once within the past year. A multiple linear regression was performed, controlling for the demographic variables which were found to be significantly related to ED use (gender and student status). The resulting ANOVA showed that energy drink users had a significantly higher risky behavior score $(M=$ 7.06, $S D=3.74)$ as compared to non-users $(M=$ $5.50, S D=2.93)(p=0.00)$.

\section{Discussion}

The current study examined the difference between energy drink users and non-users on their participation in other risky behaviors (e.g., tobacco use, illicit drug use, driving without a seatbelt) among college students. Nearly two thirds of respondents reported using EDNAs at least once within the past year. These findings were similar to previous studies reporting approximately half of college students having ever consumed an EDNA (Malinauskas et al., 2007, O’Brien et al., 2008). A growing trend in the literature has been observed in the consumption of EDA (Malinauskas et al., 2007,
O'Brien et al., 2008). Consistent with previous studies, EDA and EDNA consumption is high and is therefore important to study.

Consumption of EDAs includes risks including severe dehydration and a perception that one is less drunk than he or she really is (Thombs et al., 2008). Although in the current study only about a quarter of participants used EDAs within the past year, $87.5 \%$ of EDNAs also reported using alcohol within the past year. As indicated by recent research, this may indicate an increased likelihood that at some point these individuals would mix the two substances together, posing an increased health risk. Arria, Caldeira, Kasperski, O’Grady, and Vincent (2011) found that college students who reported being high-frequency EDNA users were twice as likely to classify as alcohol dependent as compared to non-users and low frequency users, while another study discovered that those who expressed positive attitudes toward EDA consumption also exhibited a high risk for EDA use and heavy episodic drinking (Varvil-Weld, Marzell, Turrisi, Mallett, \& Cleveland, 2013).

Students who had consumed EDNAs reported they did so for such reasons as feeling tired from insufficient sleep, to increase overall energy, to stay awake to study or finish a project, and to stay awake for class. Since these top reasons seemed to be related to inadequate sleep and the need for increased energy, possible strategies to reduce the perceived 'need' for EDNA consumption among college students should be employed. College health educators might work individually with students or within groups to strategize ways to improve sleeping habits, time management, and/or stress management. To the authors' knowledge, no published research exists which examines the effectiveness of any interventions or prevention efforts related to EDNA use among college students.

The most common reasons students reported consuming EDAs were to get drunk, to stay awake longer for partying, because a friend offered it, to drink prior to going to bars, and to see what would happen. College students seem to consume EDAs primarily for the enhanced effects of alcohol, and to prolong partying and 
socializing. Because these reasons are so closely tied to enhancing the effects of alcohol, this lends to the potential benefits of combining EDNA education with alcohol prevention programming among college students. As with EDNA use, no known published research exists which investigates prevention strategies for EDA use among college students.

In addition, the reported reasons for consuming EDAs seem to be tied to social influence (e.g., tried the beverage because a friend offered it). Almost $70 \%$ of the participants reported having at least one or more friends who regularly consume EDAs, thereby supporting the notion of peer and social influence. However, only 38.2\% of participants in the current study reported having ever consuming an EDA, thus indicating the possibility that the individuals' perception of the norm in regard to using the beverages was much higher. Social norming campaigns may be an effective strategy in reducing ED consumption, as the practice has been shown to decrease alcohol use in college students (Perkins, Linkenbach, Lewis, \& Neighbors, 2010; Turner, Perkins, \& Bauerle, 2008).

Results indicated that energy drink users had a significantly higher mean risky behavior score as compared to non-users. Findings from the current study provide preliminary evidence that ED consumption may coincide with or be a predictor of other risky behaviors. Engaging in risky behaviors, including substance use and abuse, has been shown to negatively impact college students' mental and physical health, along with academic performance (Ahearn, 2009). It is important for future studies to investigate the connection between ED use and participation in other risky behaviors, as well as college students' perceptions and attitudes toward the consumption of EDs. It is possible that college students may perceive EDs to be less destructive than they really are, leading to increased usage and thus, greater participation in other risky behaviors.

\section{Limitations}

There were several limitations to this study. First, a convenience sample of college students was utilized, so the sample may not be representative of the university and/or may not generalize to other college campuses. The overall response rate $(14.4 \%)$ had to be estimated, since course instructors were not required to send students the survey (there was not a clear number of how many students actually received the survey). Since average response rates for web-based surveys are approximately $40 \%$, the response rate for the current study would be considered low (Archer, 2008). In addition, all data was self-report; one limitation of self-report data is social desirability bias, in which respondents answer in a socially acceptable manner (Miller, 2011). Future studies could be strengthened by including measures which evaluate respondents' tendencies to give socially desirable responses, and then statistically control for these responses (Paulhus, 1991). Another source of bias for self-report data includes selective recall, wherein some events prove to be more easily remembered than others (Fadnes, Taube, \& Tylleskar, 2008).

Limitations were also present in classification of energy drinks and their users. EDNAs were not explicitly defined on the survey. Therefore, some students may have been confused as to what constituted an EDNA. In addition, energy drink users were classified as those students who had consumed an EDNA or an EDA at least 6 times within the past year. This decision to classify energy drink users in this way was not made based on literature (none available), but rather in order to elicit a larger sample of energy drink users than those who had only consumed an EDNA or an EDA once within the past year. Future researchers may want to examine differences in risky behavior participation among users who consume EDNAs as compared to EDAs. Finally, there are limitations on what conclusions can be drawn to explain whether ED use causes participation of other risky behaviors. A longitudinal study is needed to determine whether ED use precedes other risky behaviors, or the opposite is the case.

\section{Implications}

The current study determined ED use is associated with other risky behaviors among college students, but does not indicate a causal relationship. Hence, it would be important for 
future studies to further investigate whether certain risky behaviors co-occur more often with ED consumption, and to determine potential risk factors for ED consumption. Gaining more insight into these topics would further guide health promotion programs on college campuses, which ultimately may need to target multiple risk factors. The evaluation of prevention and intervention strategies for ED consumption should also be performed, given the related health consequences and the lack of research in this area.

The findings from this study also lend themselves to future research informing policies related to the availability of EDs on and around college campuses. Campus-wide policies restricting certain negative health behaviors such as tobacco use have been shown to be successful among college students (Fallin, Roditis, \& Glantz, 2014). Therefore, policy change may be a strategy which could be effective in decreasing ED use as compared to more individualized interventions. Additional research on this topic is needed, so that evidence-based public health strategies may be employed to reduce or eliminate ED consumption among college students.

\section{References}

Ahearn, N. (2009). Risky behavior in adolescent college students. Journal of Psychological Nursing, 47(4), 21-25.

Archer, T.M. (2008). Response rates to expect from web-based surveys and what to do about it. Journal of Extension, 46(3). Retrieved from http://www.joe.org/joe/2008june/rb3.php

Arria, A., Caldeira, K.M., Kasperski, S.J., Vincent, K.B., Griffiths, R.R., O’Grady, K.E. (2011). Energy drink consumption and increased risk for alcohol dependence. Alcoholism: Clinical and Experimental Research, 35, 365-375.

Arria, A., \& O’Brien, M. (2011). The 'high’ risk of energy drinks. Journal of the American Medical Association, 305(6), 600-601.

Ajzen, I. (1991). The theory of planned behavior. Organizational Behavior and Human Decision Processes, 50, 179-211

Center for Science in the Public Interest. (2012). Caffeine content in food and drugs. Retrieved from http://www.cspinet.org/new/cafchart.htm

Centers for Disease Control and Prevention. (2014). Fact sheets - caffeine and alcohol. Retrieved from http://www.cdc.gov/alcohol/fact-sheets/cab.htm

Core Institute. (2012). The Core Alcohol and Drug Survey. Retrieved from http://www.core.siuc.edu

DiClemente, R.J., Crosby, R.A., \& Kegler, M.C. (2002). Emerging Theories in Health Promotion Practice and Research. San Francisco: Jossey-Bass.

Fadnes, I., Taube, I., \& Tylleskar, T. (2008). How to identify information bias due to self-reporting in epidemiological research. The Internet Journal of Epidemiology, 7(2), 1-10.

Fallin, A., Roditis, M., \& Glantz, S.A. (2014) Association of campus tobacco policies with secondhand smoke exposure, intention to smoke on campus, and attitudes about outdoor smoking restrictions. American Journal of Public Health. Advance online publication. doi:10.2105/AJPH.2014.302251

Ferreira, S., Túlio de Mello, M., Pompéia, S., \& Oliveira de Souza-Formigoni, M. (2006). Effects of energy drink ingestion on alcohol intoxication. Alcoholism: Clinical and Experimental Research, 30(4), 598-605.

Fitzpatrick, L. (2007). The newest addictions on campus. Time Magazine, August 12. Retrieved from http://www.time.com/time/specials/2007/article/0,28804,1651473_1651472_1651348,00.html

Forke, C.M., Myers, R.K., Catallozzi, M. \& Schwarz, D.F. (2008). Relationship violence among female and male college undergraduate students. Archives of Pediatric and Adolescent Medicine, 162(7), 634-641.

IBM. (2011). SPSS (Version 20.0) [Software]. 
Macan, T., Dipboye, R., Shahani, C., \& Phillips, A. (1990). College students' time management: Correlations with academic performance and stress. Journal of Educational Psychology, 82(4), 760-768.

Malinauskas, B., Aeby, V., Overton, R., Carpenter-Aeby, T., \& Barber-Heidal, K. (2007). A survey of energy drink consumption patterns among college students. Nutrition Journal, 6(35), 1-7.

Miller, A. (2011). Investigating social desirability bias in student self-report surveys. Retrieved from http://cpr.iub.edu/uploads/SDB.pdf

Miller, K. (2008). Energy drinks, race, and problem behaviors among college students. Journal of Adolescent Health, 43(5), 490-497.

National Eating Disorders Association. (2013). Collegiate survey project. Retrieved from http://www.nationaleatingdisorders.org/CollegiateSurveyProject

National Institutes of Health. (2013). College drinking. Retrieved from http://www.niaaa.nih.gov/alcoholhealth/special-populations-co-occurring-disorders/college-drinking

O’Brien, M., McCoy, T., Rhodes, S., Wagoner, A., \& Wolfson, M. (2008). Caffeinated cocktails: Energy drink consumption, high-risk drinking, and alcohol-related consequences among college students. Academic Emergency Medicine, 15(5), 453-460.

Paulhus, D.L. (1991). Measurement and control of response bias. In J.P. Robinson, P.R. Shaver, \& L.S. Wrightman (Eds.). Measurement and control of response bias. Measures of personality and social psychological attitudes. New York: Academic Press.

Perkins, H.W., Linkenbach, J.W., Lewis, M.A., \& Neighbors, C. (2010). Effectiveness of social norms media marketing in reducing drinking and driving: A statewide campaign. Addictive Behaviors, 35, 866-874.

Reissig, C., Strain, E., \& Griffiths, R. (2008). Caffeinated energy drinks: A growing problem. Drug and Alcohol Dependence, 99(1-3), 1-10.

Smith, N. \& Atroch, A.L. (2010). Guarana's journey from regional tonic to aphrodisiac and global energy drink. Evidence Based Complementary and Alternative Medicine, 7(3), 279-282.

Steinberg, L., Albert, D., Cauffman, E. Banich, M., Graham, S., Woolard, J. (2008). Age differences in sensation seeking and impulsivity as indexed by behavior and self-report: evidence for a dual systems model. Developmental Psychology, 44(6), 1764-1768.

Substance Abuse and Mental Health Services Association [SAMHSA]. (2014). 1 in 10 energy drinkrelated emergency department visits results in hospitalization. Retrieved from http://beta.samhsa.gov/data/sites/default/files/spot124-energy-drinks-2014.pdf

Thombs, D., O’Mara, R., Tsukamoto, M., Rossheim, M., Weiler, R., Merves, M., \& Goldberger, B. (2008). Event-level analyses of energy drink consumption and alcohol intoxication in bar patrons. Addictive Behaviors, 35(10), 325-330.

Traue, J. \& Stahlman, S. (n.d.). Alcohol mixed with high levels of caffeine: What campus professionals need to know. Retrieved from http://www2.myacpa.org/docs/publications/ACPA_Alcohol_Caffeine.pdf

Turner, J., Perkins, H.W., \& Bauerle, J. (2008). Declining negative consequences related to alcohol misuse among college students exposed to social norms marketing intervention on a college campus. Journal of American College Health, 57, 85-93.

U.S. Department of Education [USDE]. (2010). Prevention update: Energy drinks. Retrieved from http://www.higheredcenter.org/files/prevention_updates/june2010.pdf

U.S. Food and Drug Administration [FDA]. (2010). FDA warning letters issued to four makers of caffeinated alcoholic beverages [Press release]. Retrieved from http://www.fda.gov/NewsEvents/Newsroom/PressAnnouncements/ucm234109.htm

Varvil-Weld, L., Marzell, M., Turrisi, R., Mallett, K.A., \& Cleveland, M.J. (2013). Examining the relationship between alcohol-energy drink risk profiles and high-risk drinking behaviors. Alcoholism: Clinical and Experimental Research, 37(8), 1410-1416.

Zuckerman, M. (1979). Sensation seeking and risk taking. In C.E. Izard (Ed.). Emotions in personality and psychopathology. New York: Pienum. 
Author Information

*Julia Buchanan, M.S.

Department of Educational Leadership Studies, University of Kentucky.

Contact: julia.buchanan@uky.edu

Melinda Ickes, Ph.D.

Department of Kinesiology and Health Promotion, University of Kentucky.

Contact: Melinda.ickes@uky.edu

* corresponding author 